\title{
Inmunoexpresión de biomarcadores Bax, Bcl-2, CD- 138, H3, Ki-67, MCM3 y p53 en liquen plano oral
}

\author{
Immunoexpression of biomarkers Bax, BCl-2, Cd-138, \\ $\mathrm{H} 3, \mathrm{Ki}-67, \mathrm{Mcm} 3$ and $p 53$ in oral lichen planus
}

\author{
Lucía Dávila' ORCID: 0000-0002-4641-7229 \\ Tatiana Suarez' ORCID: 0000-0002-4234-6048 \\ Vanesa Pereira-Prado ${ }^{1}$ ORCID: 0000-0001-7747-671 \\ Gabriela Vigil' ORCID: 0000-0002-0617-1279 \\ Ramiro Tomasi² ORCID: 0000-003-2705-8221 \\ Gabriel Tapia ${ }^{3}$ ORCID: 0000-0003-4563-9142 \\ Ruben Del Muro Delgad0 ${ }^{4}$ ORCID: 0000-0003-3169-2962 \\ Ronell Bologna-Molina5 ORCID: 0000-0001-9755-4779
}

\section{Resumen}

El objetivo del presente trabajo fue determinar la expresión de diversos biomarcadores moleculares en liquen plano oral para ayudar a comprender su conducta biológica. Materiales y métodos: Se realizó un estudio inmunohistoquímico en 40 casos de liquen plano oral contra BAX, BCL-2, CD-138, Histona 3, Ki-67, MCM3 y p53, en el Área de Patología Molecular Estomatológica de la Facultad de Odontología, UDELAR, Uruguay. Resultados: Se observó mayor expresión de BAX en contraposición con BCL-2, sugiriendo un comportamiento proapoptótico, respaldado a su vez por la ausencia de expresión de p53. La expresión de los marcadores de proliferación celular fue en todo el tejido lesional observado, sugiriendo así alteraciones de la proliferación. CD-138 se expresó de manera intensa y uniforme, determinando una baja alteración de las uniones intercelulares para estos casos. Conclusiones: La alteración en la expresión de las proteínas estudiadas sugiere un trastorno en los mecanismos proliferativos y apoptóticos, los cuales se asocian con una conducta patológica de la mucosa oral.

Palabras clave: liquen plano oral, apoptosis, proliferación.

\footnotetext{
Área de Patología Molecular Estomatológica. Facultad de Odontología, Universidad de la República, Uruguay.

Cátedra de Anatomía Patológica A. Facultad de Odontología, Universidad Nacional de Córdoba, Argentina.

Cátedra de Histología. Facultad de Odontología, Universidad de la República, Uruguay.

Departamento de Sistemas Biológicos. División de Ciencias de la Salud. Universidad Autónoma Metropolitana, México.

Área de Patología Molecular Estomatológica. Facultad de Odontología, Universidad de la República, Uruguay.
}

Fecha de recibido: 06.05.2019 - Fecha de aceptado: 29.07.2019 


\section{Abstract}

This study aims to establish an association of the expression of specific biomarkers in oral lichen planus to understand its biological behavior. Materials and methods: An immunohistochemistry study was conducted in 40 cases of oral lichen planus against BAX, BCL-2, CD138, Histone 3, Ki-67, MCM3 and p53 at the Molecular Pathology Area of the School of Dentistry, UDELAR, Uruguay. Results: A greater expression of BAX was detected compared to BCL-2, suggesting a pro-apoptotic behavior, supported by the absence of p53 expression. MCM3 expression was more sensitive than Ki-67, considering proliferation alterations. CD-138 had a more intense and uniform expression, determining fewer intercellular adhesion alterations. Conclusions: The expression of the proteins studied suggests an alteration in proliferative and apoptotic mechanisms, associated with a pathological behavior of the oral mucosa.

Keywords: oral lichen planus, apoptosis, proliferation.

\section{Introducción}

El liquen plano es una enfermedad inflamatoria, mediada inmunológicamente, que afecta al epitelio escamoso estratificado, que se manifiesta en piel y mucosas ${ }^{(1)}$. La primera descripción fue realizada por Erasmus Wilson en 1869; etimológicamente liquen plano proviene del vocablo griego leichen "musgo de árbol" y del latín planus "plano" por el aspecto de las lesiones ${ }^{(2)}$. Afecta al 0,2-1,9\% de la población, encontrándose principalmente en edades entre los 30 y 70 años ${ }^{(3)}$. Mientras que el liquen de piel es auto

\section{Resumo}

O objetivo deste trabalho foi determinar a expressão de vários biomarcadores moleculares no líquen plano oral para ajudar a compreender seu comportamento biológico. Materiais e métodos: Foi realizado um estudo imunohistoquímico em 40 casos de líquen plano oral contra BAX, BCL-2, CD-138, Histona 3, Ki-67, MCM3 e p53, na área de Patologia Molecular Estomatológica da Faculdade de Odontologia , UDELAR, Uruguai. Resultados: Observou-se aumento da expressão de BAX em contraste com BCL-2, sugerindo um comportamento proapoptótico, apoiado por sua vez pela ausência da expressão de p 53. A expressão de marcadores de proliferação celular foi observada em todo o tecido da lesão, sugerindo alteraçôes na proliferação. CD-138 foi expressado de maneira intensa e uniforme, determinando uma baixa alteração das junções intercelulares para esses casos. Conclusôes: A alteração na expressão das proteínas estudadas sugere um distúrbio nos mecanismos proliferativos e apoptóticos, os quais estáo associados a um comportamento patológico da mucosa oral.

Palavras-chave: líquen plano oral, apoptose, proliferação.

limitado, el liquen plano oral (LPO) es crónico, no suele remitir espontáneamente y es considerado como potencialmente maligno según la Organización Mundial de la Salud (4), estableciendo este riesgo de transformación maligna a cáncer oral de células escamosas por debajo del $1 \%{ }^{(5)}$.

En cuanto a sus características clínicas, es una lesión que se localiza con mayor frecuencia en mucosa yugal, seguida por la lengua (márgenes laterales), dorso de lengua y encía. El LPO se puede encontrar en diferentes presentaciones clínicas, de las cuales se distinguen el liquen 
plano reticular, caracterizado por tener un patrón de estrías blanquecinas (queratinización) de 0,1 a $2 \mathrm{~mm}$ de ancho; liquen plano atrófico en el cual se observa rojizo, con adelgazamiento del epitelio; liquen plano ulcerado definido por la pérdida de superficie epitelial, formando ulceraciones irregulares cubiertas de fibrina, rodeadas de áreas atróficas; liquen plano a placas, conforma áreas sólidas de queratinización (lengua o mucosa yugal mayoritariamente); gingivitis descamativa, afecta la encía adherida, presentándose inflamada, lisa y brillante; liquen plano ampolloso se observa cuando existe una separación del epitelio, posterior a la pérdida de células basales ${ }^{(5-7)}$.

Desde el punto de vista histopatológico el LPO puede presentar diversas alteraciones como hiperqueratosis (orto o paraqueratosis), acantosis, granulosis, espongiosis, cuerpos coloides, exocitosis linfocitaria y atrofia epitelial, siendo esencial la degeneración vacuolizante de la capa basal del epitelio y el infiltrado inflamatorio subyacente en banda ${ }^{(8)}$.

La etiopatogénesis del LPO no está completamente dilucidada, pero se cree que posiblemente se deba a una reacción autoinmune mediada por células $\mathrm{T}$ citotóxicas (CD8+) contra los queratinocitos basales, inducidos por un cambio antigénico en la piel, o la mucosa ${ }^{(9)}$. La combinación de mecanismos específicos e inespecíficos parecen causar la acumulación de linfocitos $\mathrm{T}$ en la lámina propia subyacente al epitelio, la ruptura de la lámina basal, la migración de los linfocitos $\mathrm{T}$ intraepiteliales $\mathrm{y}$ la apoptosis de los queratinocitos ${ }^{(10)}$. Los antígenos alterados de los queratinocitos basales son reconocidos por las células de Langerhans y presentados a los linfocitos T CD4+ localmente o a través de su paso por los nódulos linfáticos por medio del HLA II (Antígeno Leucocitario Humano II), desencadenando su expansión clonal. Después de ser activadas las células T se diferencian en dos subtipos funcionales: células T tipo 1 (Th1) que producen TNFy (Factor de Necrosis tumoral $\gamma$ ) y células T tipo 2 (Th2) que producen diferentes interleuquinas (IL): IL-4, IL-5, IL-6, IL-10 y IL-13(11). El TNF estimula la proliferación acelerada de los queratinocitos basales y la subsecuente acumulación de queratina superficial que clínicamente se observa como hiperqueratosis, bajo este estímulo aumentan las capas celulares en los diferentes estratos y la descamación epitelial se hace más lenta. Debido a la intensa proliferación basal se producen alteraciones intracelulares que son percibidas por los linfocitos T CD8+ intraepiteliales a través de la expresión de HLA I por los queratinocitos basales y se activa la apoptosis en el estrato basal ${ }^{(12)}$.

Las mutaciones y alteraciones moleculares presentes en LPO podrían jugar un papel decisivo en la transformación neoplásica ${ }^{(13)}$.

Firzpatrick, describe que los LPO erosivos mostraron un mayor riesgo de transformación neoplásica ${ }^{(14)}$.

Para el diagnóstico de LPO se utilizan frecuentemente los criterios clinicopatológicos de la OMS los cuales consisten en, presencia de lesiones bilaterales, mayoritariamente simétricas; estriado blanco retículo-papular; lesiones erosivas, atróficas, ampollares y en placas con la presencia en otra zona de la cavidad oral de estrías blancas. Los criterios histopatológicos se basan en la presencia de infiltrado yuxtaepitelial en banda, constituido principalmente por linfocitos; signos de degeneración hidrópica del estrato basal; y ausencia de displasia epitelial ${ }^{(15)}$. El tratamiento de LPO generalmente consiste en aplicaciones tópica y/o sistémica con corticosteroides ${ }^{(5)}$.

El estudio de diversos biomarcadores, tanto de proliferación celular (Ki-67, MCM3, Histona 3), de supresión tumoral (p53), y de regulación del proceso de apoptosis (BCL-2, BAX) pueden ayudar a establecer una posible asociación de la expresión de estas proteínas con el comportamiento patológico del LPO. Por lo tanto, el objetivo del presente estudio consistió únicamente en determinar y correlacionar morfológicamente la presencia de estas proteínas con el LPO 
para generar información que ayude a comprender la conducta biológica de esta lesión.

\section{Materiales y métodos}

Fueron incluidos un total de 40 casos de LPO, de muestras provenientes de la Cátedra de $\mathrm{Pa}$ tología de la Universidad Autónoma Metropolitana (UAM, Ciudad de México, México) y la Universidad de Córdoba, Argentina, diagnosticadas durante el periodo comprendido del 2008 a inicios de 2013. Los especímenes se encontraban conservados en bloques de parafina. Se realizaron 7 cortes de los tejidos a 4 micras para la técnica de inmunohistoquímica y 1 corte para tinción con hematoxilina y eosina. El estudio morfológico se realizó por dos patólogos con experiencia en LPO tomando en cuenta parámetros histopatológicos preestablecidos y con las características clínicas de la lesión (16). Los estudios de inmunohistoquímica se realizaron en el laboratorio de Patología Molecular Estomatológica, en la Facultad de Odontología, Universidad de la República (UDELAR, Montevideo, Uruguay). Este estudio fue aprobado por el Comité de Ética de la Facultad de Odontología, UDELAR, expediente: 120/16.

Se hicieron cortes de $4 \mu \mathrm{m}$ de espesor, los cuales se montaron sobre laminillas tratadas con poly-L-lisina, posteriormente los cortes fueron desparafinados en una estufa a $60^{\circ} \mathrm{C}$ por $30 \mathrm{mi}$ nutos y se colocaron en xilol por 5 minutos. Los cortes se hidrataron en un tren de concentraciones decrecientes de alcoholes (absoluto, 90, 80,70 y $50 \%$ ) y enjuagues con agua destilada. Para el desenmascaramiento de los epítopes se realizó recuperación antigénica con solución de citrato de sodio con $\mathrm{pH} 6$, dicha recuperación se hace en una olla de presión dentro del microondas con potencia máxima de 750 w. Las peroxidasas endógenas fueron bloqueadas con peróxido de hidrógeno al 0,9\%, seguidas por lavados con agua destilada y solución salina amortiguada de fosfatos $\mathrm{pH}$ 7,4 (PBS). Los anticuerpos primarios se incubaron por un tiem- po de 60 minutos contra: p53 (dilución: 1:200, Bio SB/D07), MCM3 (dilución: 1:100, Leica/ DCS-141.1), Ki-67 (dilución: 1:100, DAKO/ MIB1), CD-138 (dilución: 1:100, DAKO/ MI15), BCL-2 (dilución: 1:100, Biocare/100/ D5), BAX (dilución: 1:200, DAKO Corp, Carpinteria, CA, USA, Policlonal), histona H3 (dilución: 1:50, phospho-histone H3 Genetex 300095). Posteriormente, los cortes se incubaron con el segundo anticuerpo biotinilado anti ratón/anti conejo y con el complejo streptavidina/peroxidasa (LSA-B+Dako Corporation, Carpinteria CA, USA) por 30 minutos cada uno. Los productos de la reacción se visualizaron con sustrato de 3,3'- diaminobenzidina H2O2 (Dako Corporation, Carpinteria, CA, USA). Como controles positivos se utilizaron testigos de diferentes punch de tejidos y como controles negativos se omitió la incubación con los anticuerpos primarios.

La cuantificación citoplasmática y/o membranosa se realizó visualmente utilizando un microscopio óptico (Eclipse CI-L, Nikon, Japón) amplificado a 40x, tomando en cuenta la siguiente escala semicuantitativa: 0-4 \% "tinción negativa"; $5-25 \%$ se considera una "tinción débil"; 26-50\% corresponde a una "tinción moderada” y 51-100\% corresponde a una "tinción intensa" de células. La cuantificación nuclear se determinó dividiendo el total de células positivas entre el total de células presentes en el campo de estudio y multiplicando por 100 para obtener un porcentaje; y con la ayuda de una cámara digital (Olympus C-7070) se tomaron microfotografías de todos los marcadores, de tres campos seleccionados en donde se observó mayor área de daño epitelial por respuesta inflamatoria (correspondientes a la capa basal e infiltrado subepitelial).

Los resultados se compararon subjetivamente con el tejido sano no alterado de la muestra estudiada, cuando la cantidad de material lo permitió. Usando esto como control interno. En el caso de no existir tejido disponible para 
comparación se usó como referencia tejido sano de otra muestra de mucosa.

Dado que el objetivo del estudio fue solamente evaluar presencia de las proteínas de interés a nivel morfológico, no se asociaron características clínicas al estudio.

\section{Resultados}

La expresión de marcadores relacionados con la apoptosis como ser el caso de BAX resultó predominantemente débil para el 50\% de las muestras, localizado en la capa basal del epitelio, observándose en el citoplasma de los queratinocitos basales (Fig. 1.A), mientras que BCL-2 tuvo una expresión citoplasmática débil con el $42,86 \%$ de los casos, en estratos suprabasales, alejado de la zona de ataque linfocitario; se constató expresión nuclear predominantemente negativa de $\mathrm{p} 53$, ya que el $81,4 \%$ de las muestras mostraron un promedio de positividad menor al $4 \%$ y mientras que el $18,60 \%$ restante tuvo una presencia débil de p53 en el infiltrado en banda subepitelial, esto podría sugerir actividad apoptótica en estas zonas del tejido, así como la presencia de daño celular en la capa basal, y se explicaría su expresión débil por la rápida proteólisis de esta proteína, así como su intervención únicamente en la fase G1 del ciclo celular (Fig. 1. B).

El índice promedio de proliferación celular a nivel nuclear para Ki-67 fue del 12,40\% las células proliferativas se observaron mayoritariamente en la capa basal del epitelio (Fig. 1. C), mientras que MCM3 presentó una positividad nuclear moderada a intensa en casi todos los casos con un promedio de proliferación celular del 33.36\% también en los queratinocitos basales (Fig. 1. D); por su parte los casos mostraron positividad a nivel nuclear para histona $\mathrm{H} 3$ (H3) predominantemente débil, seguida por moderada, con un promedio final de índice de proliferación celular del $26.76 \%$ y de igual manera evidenciándose en la capa basal (Fig. 1. E).

CD-138 se observó en su mayoría intensamente positivo con el 95,35\% a nivel de la membrana celular en todo el epitelio, sin expresión negativa en ninguno de los casos (Fig. 1. F).

Las lesiones mostraron un índice de proliferación activo en los queratinocitos de la capa basal y suprabasal, un leve aumento en las proteínas proapoptóticas, en capa basal e infiltrado inflamatorio subepitelial (Tabla 1).

Tabla 1. Resultados correspondientes a cada biomarcador.

\begin{tabular}{|c|c|c|c|c|c|}
\hline \multirow{2}{*}{ Biomarcadores } & \multicolumn{5}{|c|}{ Expresión (\%) } \\
\hline & $0-4$ (negativo) & 5-25 (débil) & 26-50 (moderada) & $51-100$ (intensa) & Localización \\
\hline BAX & $40,38 \%$ & $50,00 \%$ & $9,52 \%$ & $0,00 \%$ & Queratinocitos basales \\
\hline $\mathrm{BCL}-2$ & $57,14 \%$ & $42,86 \%$ & $0,00 \%$ & $0,00 \%$ & Queratinocitos basales \\
\hline p53 & $81,40 \%$ & $18,60 \%$ & $0,00 \%$ & $0,00 \%$ & Infiltrado inflamatorio subepitelial \\
\hline Ki-67 & $7,32 \%$ & $92,68 \%$ & $0,00 \%$ & $0,00 \%$ & Queratinocitos basales \\
\hline MCM3 & $8,12 \%$ & $2,70 \%$ & $45,94 \%$ & $43,24 \%$ & Queratinocitos basales \\
\hline H3 & $15,63 \%$ & $58,14 \%$ & $30,23 \%$ & $0,00 \%$ & Queratinocitos basales \\
\hline CD-138 & $0,00 \%$ & $4,65 \%$ & $0,00 \%$ & $95,35 \%$ & Queratinocitos basales y suprabasales \\
\hline
\end{tabular}




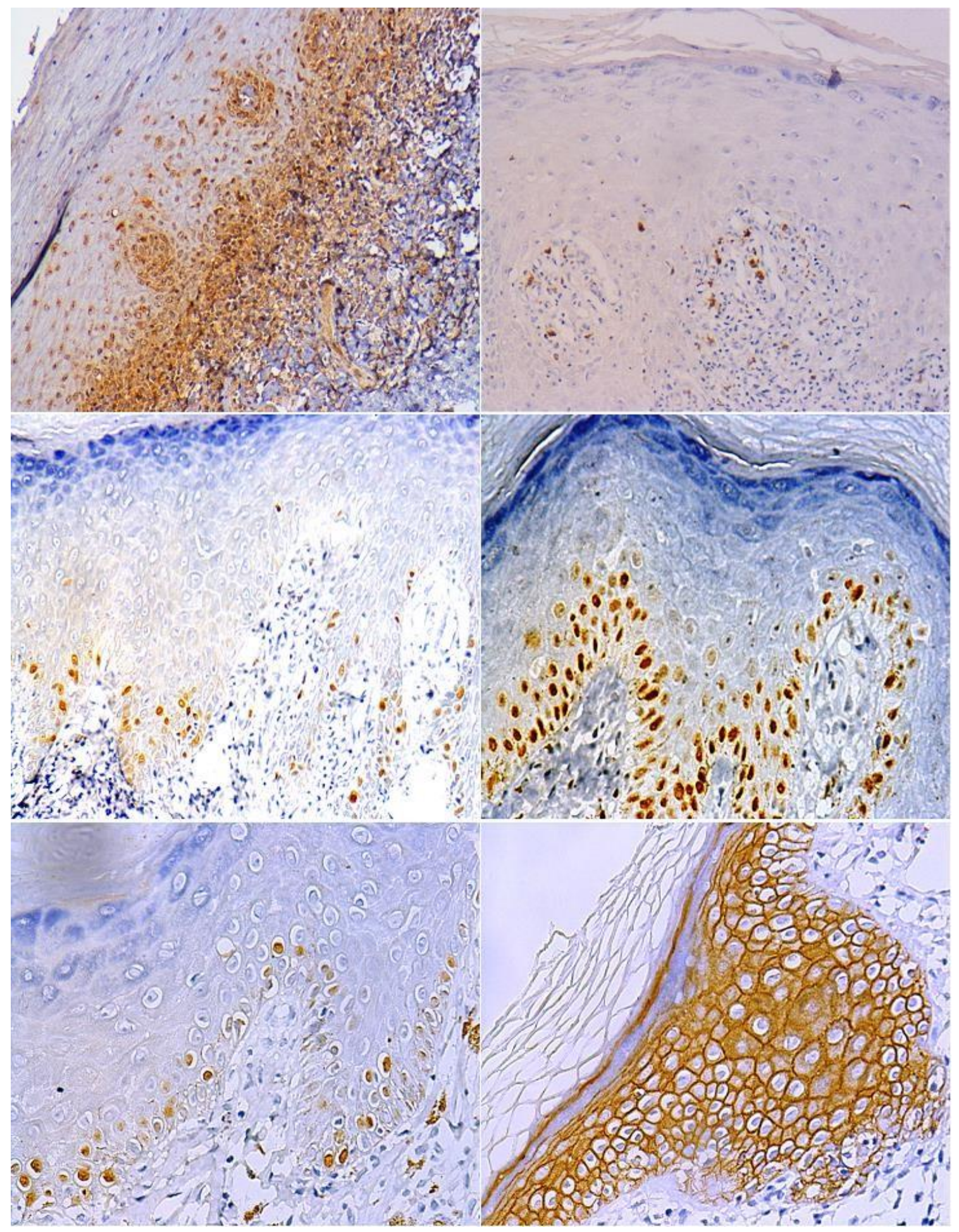

Fig. 1: Inmunoexpresión de biomarcadores en LPO. A) Expresión citoplasmática de BAX en queratinocitos de la capa basal. Aumento 40x. B) Expresión citoplasmática de p53 en el infiltrado inflamatorio subepitelial. Aumento 20x. C) Expresión nuclear de Ki-67 en queratinocitos de la capa basal. Aumento 20x. D) Expresión nuclear de MCM3 en células de la capa basal del epitelio. Aumento 20x. E) Expresión nuclear de $\mathrm{H} 3$ en queratinocitos basales. Aumento 20x. F) Expresión membranal / citoplasmática de CD-138 en todos los estratos epiteliales. 


\section{Discusión}

BAX es considerado un inductor de apoptosis, perteneciente a la familia BCL-2 ${ }^{(17)}$. La misma está constituida por una red de interacciones proteicas que regulan la apoptosis a través de la permeabilización de la membrana externa mitocondrial; BAX es translocada hacia la mitocondria, donde se activa e integra a la membrana causando la salida al citoplasma de una serie de proteínas consideradas factores apoptóticos (Fig. 2), (Citocromo c, Smac/Diablo, Omi/ HtrA2, Endonucleasa G, factor inductor de la apoptosis) ${ }^{(18)}$. Su expresión en altas cantidades se encuentra asociada con un pronóstico favorable en varios tipos de cáncer ${ }^{(19-20)}$.

El oncogén BCL-2 codifica para una proteína que bloquea un paso determinado en las vías de apoptosis (Fig. 2). Su expresión anormal, generalmente sobre expresada en células tumorales, contribuye a la expansión del daño celular y reducción de la apoptosis; al permitir la sobrevida celular, facilita la adquisición de mutaciones y transformaciones malignas (21). Su función se basa en impedir la salida de citocromo c a través de la membrana externa mitocondrial, por la formación de heterodímeros con moléculas proapoptóticas como BAX ${ }^{(22)}$. La expresión aumentada de BCL-2 en un estudio realizado sobre cáncer de células escamosas de laringe, se asoció a un estado avanzado de la enfermedad ${ }^{(23)}$.

En cuanto a la expresión de marcadores de apoptosis (BAX y BCL-2) y de detención del ciclo celular (p53) en LPO, nuestros resultados fueron muy similares a los reportados en la literatura internacional; Bascones et al., encontró en áreas lesionadas que la expresión fue leve a moderada en la capa basal, y disminuyendo en supra basal de leve intensidad o negatividad ${ }^{(24)}$. Shailaja et al. reportaron en un estudio realizado con 30 casos de LPO, inmunoexpresión negativa para BAX en un $43,3 \%$ de los casos y moderada en un 33,3\%. Mientras que para BCL- 2 la inmunoexpresión fue moderada en un $36,7 \%$ de los casos y negativa en un $26,7 \%{ }^{(25)}$. Según investigaciones de Bogdan et al. y Calenic ${ }^{(27)}$ et al. la expresión de BCL-2 no mostró diferencias significativas en comparación con el grupo control; a diferencia de la inmunoexpresión de BAX la cual fue significativamente mayor en comparación con el grupo control en ambos estudios (26-27); por otra parte, el estudio de Shailaja muestra resultados similares en cuanto a la expresión de BAX, en comparación con nuestro estudio ${ }^{(25)}$.

La función fisiológica de p53 es prevenir la acumulación de daño genético celular, ya sea por reparación previa a la división celular o causando apoptosis. Su alteración produciría por lo tanto un crecimiento celular no controlado ${ }^{(28)}$. El daño funcional de p53 ha sido implicado en el desarrollo y progresión de displasia epitelial oral y carcinoma oral de células escamosas ${ }^{(29)}$. Varios estudios coinciden en que la inmunoexpresión de p53 es significativamente mayor en LPO en las capas basal y suprabasal cuando se compara con un grupo control (mucosa oral sana) ${ }^{(25-27,30,31)}$.

En condiciones normales p53 se encuentra en niveles bajos como resultado de su rápida proteólisis ${ }^{(27)}$. Nuestros resultados mostraron una expresión débil de esta proteína a nivel de la capa basal, sugiriendo un aumento en la apoptosis celular de la zona.

Ki-67 es una proteína nuclear que interviene en el ciclo celular, asociada a la proliferación celular y utilizada como un marcador de proliferación celular para medir el crecimiento celular tumoral ${ }^{(28)}$. Este marcador puede ser utilizado en la detección temprana de cáncer de células escamosas, expresándose en las fases G1, S, G2, $\mathrm{M}$ del ciclo celular ${ }^{(32)}$.

MCM3 forma parte de un grupo de proteínas (minichromosome maintenance proteins) relacionadas con la replicación del $\mathrm{ADN}$, teniendo su aumento un rol en el proceso de transformación maligna celular ${ }^{(25)}$. Diversos estudios sobre la expresión de MCM3 a nivel de linfomas, leucemia, carcinoma del cérvix, de mama, de riñón, estómago, pulmón, colon 

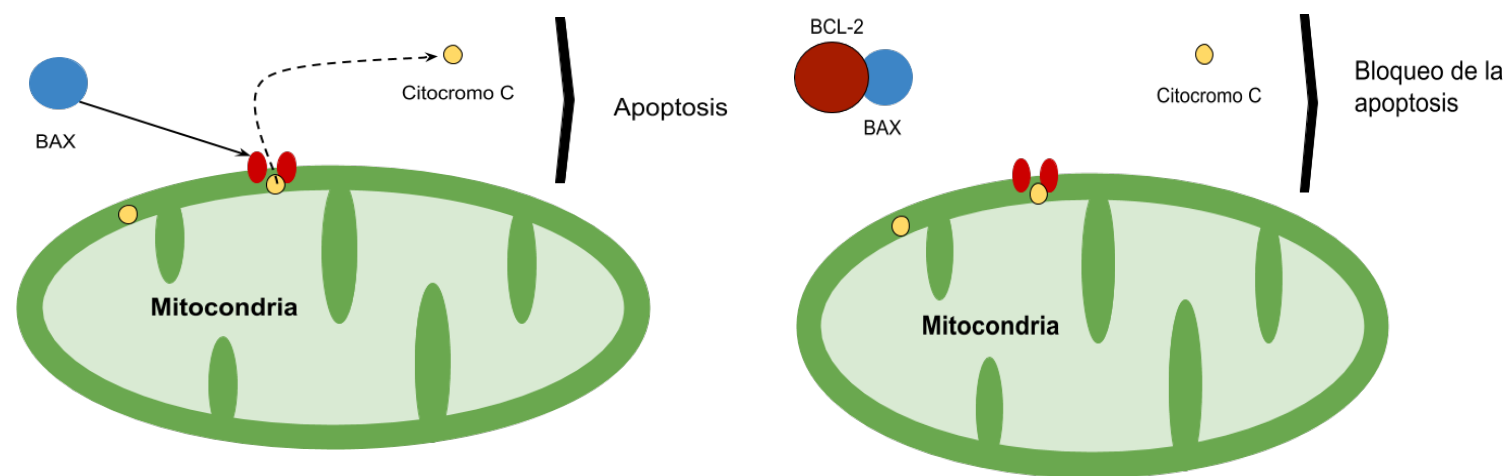

Fig. 2: El biomarcador proapoptótico BAX al unirse al receptor de membrana, provoca la salida de citocromo c de la mitocondria, llevando a la muerte de la célula; cuando BCL-2 se une a esta proteína, se bloquea esta vía de apoptosis.

y melanoma, entre otros, han determinado un incremento de su expresión asociándolo a un peor pronóstico ${ }^{(33)}$.

Ki-67 que se presenta en todas las fases activas del ciclo celular, excepto G0 y G1 temprana, mientras que MCM3 se expresa en niveles elevados durante todas las fases del ciclo celular incluyendo G1 temprana y excluyendo G0 ${ }^{(35)}$. En un estudio realizado por Rezazadeh et al., en base a la expresión de Ki-67 y MCM3 en el cáncer oral de células escamosas, MCM3 resultó positivo en el $96,4 \%$ y $\mathrm{Ki}-67$ en el $78 \%$, en comparación con los casos de mucosa normal en donde los índices de proliferación son bajos (32). La proliferación celular medida con estas dos proteínas en nuestro estudio mostró una inmunoexpresión de 92,68\% débil para Ki-67 y para MCM3 45,94\% moderada, lo que sugiere el aumento de la misma, viéndose reflejado en la expresión positiva de ambas en el LPO.

$\mathrm{H} 3$ es una de las cinco principales histonas protéicas involucradas en la estructura de la cromatina en células eucariotas ${ }^{(36)}$. Diversos estudios consideran que las variaciones y modificaciones en el estado de $\mathrm{H} 3$ juegan un rol en la regulación de los genes a largo plazo ${ }^{(37)}$. Dos genes, $\mathrm{H} 3 \mathrm{~F} 3 \mathrm{~A}$ y $\mathrm{H} 3 \mathrm{~F} 3 \mathrm{~B}$, codifican la proteína $\mathrm{H} 3$, pero ambos son regulados de forma diferente en procesos cancerígenos, considerando que una sobreexpresión de H3F3A promueve la invasión celular y la progresión del cáncer ${ }^{(38-40)}$.
Finalmente, en nuestro estudio evidenciamos que $\mathrm{H} 3$ tiene una expresión de débil a modera$\mathrm{da}$, sugiriendo una leve a nula alteración de esta proteína asociada a la patogénesis del LPO o a la conducta biológica del mismo.

Syndecan 1, (CD-138), forma parte de una familia de receptores que participan en la adhesión célula-célula y célula-matriz, localizado a nivel basal y suprabasal de las capas celulares ${ }^{(38)}$. Su expresión aumentada en la diferenciación de los queratinocitos a nivel del epitelio normal o hiperplásico se encuentra reducida en el carcinoma de células escamosas y lesiones premalignas, pudiendo tener un valor pronóstico en la determinación del resultado clínico de la lesión ${ }^{(39)}$. Según el estudio realizado por Manal et al., se determinó que CD-138 presenta una expresión positiva alta en epitelio estratificado normal, pero su expresión se vio disminuida en los casos de LPO erosivo, y se asoció la alta inmunoreactividad de CD-138 a las lesiones que no se malignizaron, considerando este marcador con posible utilidad para determinar el potencial de malignización de las lesiones ${ }^{(31)}$. En nuestro estudio pudimos apreciar que CD-138 se encontraba conservado, lo que se interpreta como una continuidad en la cohesión célulacélula.

Una limitante importante de este estudio es que no en todos los casos se pudo contrarrestar o comparar la expresión de las proteínas estudia- 
das con el tejido sano adyacente, debido a que algunas muestras no lo permitían al ser el tejido insuficiente.

\section{Conclusiones}

Se sugieren mecanismos pro- apoptóticos en la capa basal del epitelio, esto sustentado por una mayor expresión de BAX en comparación con BCL-2; esta especulación se refuerza en la presencia de p53 como único marcador observado en el infiltrado subepitelial

El trastorno de los mecanismos proliferativos se asocia al aumento de los biomarcadores $\mathrm{Ki}$ 67, MCM3 y H3, sugiriendo una respuesta de los queratinocitos basales al daño inmunitario mediado por linfocitos hacia la capa basal del epitelio,

Se sugiere a futuro ampliar el trabajo con una casuística mayor comparando con áreas sanas adyacentes o mucosa oral normal de pacientes $\sin$ LPO.

\section{Contribución de autoría}

1. Concepción y diseño del estudio

2. Adquisición de datos

3. Análisis de datos

4. Discusión de los resultados

5. Redacción del manuscrito

6. Aprobación de la versión final del manuscrito

DL y TS han contribuido en: $1,3,4,5$, y 6.

VP, GV y RB han contribuido en: 1, 2, $3,4,5$ y 6 .

TR, TG y DR han contribuido en: 2, 4 , 5 y 6.

\section{Referencias}

1. Cerero-Lapiedra R. Malignización del liquen plano oral. Av. Odontoestomatol 2008; 24 (1): 97-103.
2. Rodríguez Acar M, Carbajal Pruenda P. Liquen plano. Revisión de la literatura. Rev Cent Dermatol Pascua. 2006; 15 (3): 203-208.

3. Bermejo F, López-Jornet P. Liquen plano oral. Naturaleza, aspectos clínicos y tratamiento. RCOE. 2004; 9 (3): 395-408.

4. Warnakulasuriya S, Johnson NW, Van Der Waal I. Nomenclature and classification of potentially malignant disorders of the oral mucosa. J Oral Pathol Med. 2007; 36: 575-580.

5. Cawson R, Odell E. Fundamentos de medicinal y patología oral. 9a ed. Barcelona: Elsavier; 2017. 262-267p.

6. Blanco Carrión A, Otero Rey E, Peñamaría Mallón M, Diniz Freitas M. Diagnóstico del liquen plano oral. Av Odontoestomatol 2008; 24 (1): 11-31.

7. Sapp J.P. Eversole L.R. Wysolki G.W. Patología Oral y Maxilofacial. 2a ed. Madrid: Elsavier; 2004. 250-253p.

8. Bascones-Ilundain C, González Moles MA, Campo-Trapero J, Bascones-Martínez A. Liquen plano oral (II). Mecanismos apoptóticos y posible malignización. Av. Odontoestomatol 2006; 22 (1): 21-31.

9. Farhi D, Dupin N. Pathophysiology, etiologic factors, and clinical management of oral lichen planus, part I: facts and controversies. Clin. Dermatol. 2010; 28(1) :100-108.

10. Gorsky M, Epstein JB, Hasson-Kanfi H, Kaufman E. Smoking habits among patients diagnosed with oral lichen planus. Tobacco Induced Diseases. 2004; 2(2): 103-8.

11. Iijima W, Ohtani $H$, Nakayama $T$, Sugawara $Y$, Sato E, Nagura H, Yoshie O, Sasano T. Infiltrating CD8+ T cells in oral lichen planus predominantly express CCR 5 and CXCR 3 and carry respective chemokine ligands RANTES/CCL5 and IP-10/CXCL10 in their cytolytic granules: a potential self-recruiting mechanism. Am. J. Pathol. 2003; 163(1): 261- 268.

12. Lodi G, Scully C, Carrozzo M, Griffiths M, Sugerman PB, Thongprasom K. Current controversies in oral lichen planus: report of an international consensus meeting. Part 1. Viral infections and etiopathogenesis. Oral Surg Oral Med Oral Pathol Oral Radiol Endod. 2005; 100(1): 40-51. 
13. Sreenivasan V . The malignant potential of oral lichen planus - confusion galore. OOOO Journal.2013; 115(3): 415.

14. Fitzpatrick S, Hirsch S, Gordon S. The malignant od oral lichen planus and oral lichenoid lesions. A systematic review. JADA. 2014; 145 (1): 45-56.

15. Shivhare P, Gupta A, Yadav M, Konidena A, Shankarnarayan L. Evaluation of different diagnostic criteria of diseases manifesting the oral cavity - A review. Part-1. JOBCR. 2016; 6(2): 135-141.

16. Fernández-González F, Vázquez R, Reboiras D, Gándara P, García A, Gándara JM. Histopathological findings in oral lichen planus and their correlation with the clinical manifestations. Med. Oral Patol. Oral Cir. Bucal. 2011; 16(5): 641-646.

17. Druilhe A, Benoit W, Tsicopoulos A, Lapa JR, Tillie-Leoblond I, Tonnel A, Pretolani M. Apoptosis, proliferation, and expression of bcl2, Fas and Fas-ligand in bronchial biopsies from asthmatics. AJRCCM. 1998; 19(5): 747-57.

18. Renault T, Dejean L, Manon Stephen. A brewing understanding of the regulation of $\mathrm{Bax}$ función by $\mathrm{Bcl}-\mathrm{xL}$ and $\mathrm{Bcl}-2$. Mechanisms of Agening and Development. 2017; 161: 201210.

19. Bose P, Klimowicz AC, Kornaga E, Petrillo SK, Matthews TW, Chandarana S, Magliocco AM, Brockton NT, Dort JC. Bax expression measured by AQUanalysis is an independent prognostic marker in oral squamous cell carcinoma. BMC Cancer. 2012; 12: 332-343.

20. Sagari S, Sanadhya S, Doddamani M, Rajput R. Molecular markers in oral lichen planus: A systematic review. JOMFP. 2016; 20(1): 115121.

21. Suri C. The immunohistochemical evaluation of the expression of BCL-2 in different histological grades of squamous cell carcinoma. J Clin Diagn Res. 2009; 3:1891-9.

22. Amarante GP, Green DR. The regulation of apoptotic cell death. Braz J Med Biol Res. 1999; 32(9): 1053-1061.

23. Pruneri G, Pignataro L, Carboni N, Ronchetti D, Cesana B, Ottaviani A, Neri A, Buffa R. Clinical relevance of $\mathrm{p} 53$ and bcl-2 protein over-expression in laryngeal squamous-cell carcinoma. Int. J. Cancer. 1998; 79: 263-268.
24. Bascones-Ilundain C, González Moles MA, Campo-Trapero J, Bascones-Martínez A. Liquen plano oral (II). Mecanismos apoptóticos y posible malignización. Av Odontoestomatol. 2006; 22(1): 21-27.

25. Shailaja G, Kumar JV, Baghirath PV, Kumar U, Ashalata G, Krishna AB. Estimation of malignant transformation rate in cases of oral epithelial dysplasia and lichen planus using immunohistochemical expression of $\mathrm{Ki}-67$, p53, BCL-2, and BAX markers. DRJ. 2015; 12(3): 235-242.

26. Bogdan C, Kazuhiko O, Ken Y, Seban T, Tomoko T, Toshio I. Role of p53-mediated apoptotic pathway in oral lichen planus: Relationship among pro-apoptotic, anti-apoptotic, and keratinocytic markers. Journal of Oral and Maxillofacial Surgery, Medicine, and Pathology. 2014; 26(2): 221-227.

27. Tampa M, Caruntu C, Mitran M, Mitran C, Sarbu I, Rusu LC, Matei C, Constantin C, Neagu M, Georgescu SR. Markers of Oral Lichen Planus Malignant Transformation. Dis Markers. 2018

28. Humayun S, Prasad VR. Expression of $\mathrm{p} 53$ protein and ki-67 antigen in oral premalignant lesions and oral squamous cell carcinomas: An immunohistochemical study. Natl J Maxillofac Surg. 2011; 2(1): 38-46.

29. Ravi D, Nalinakumari KR, Rajaram RS, Nair MK, Pillai MR. Expresion of programmed cell death regulatory $\mathrm{p} 53$ and bcl-2 proteins in oral lesions. Cancer Letters. 1996; 105 (2): 139146.

30. Basheer S, Shameena PM, Shuda S, Varma S, Vidyanath S, Varekar A. Expression of survivin and p53 in oral lichen planus, lichenoid reaction and lichenoid dysplasia: An immunohistochemical study. JOMFP. 2017;21 (3): 456-457.

31. Manal M, Zyada PhD, Hala E, Fikry PhD. Immunohistochemical study of syndecan-1 downregulation and the expression of P53 protein in oral lichen planus: a clinicopathologic correlation with hepatitis $\mathrm{C}$ infection in the Egyptian population. Annals of Diagnostic Pathology. 2010; 14(3): 153-161.

32. Rezazadeh F, Ebrahimi R, Andisheh-Tabdir A, Ashraf M, Khademi B. Evaluation of the Ki67 and MCM3 Expression in Cytologic Smear 
of Oral Squamous Cell Carcinoma. J Dent (Shiraz). 2017; 18(3): 207-211.

33. Gan N, Du Y, Zhang W, Zhou J. Increase of $\mathrm{Mcm} 3$ and Mcm4 expression in cervical squamous cell carcinomas. Eur. J. Gynaeco. Oncol. 2010; 31: 291-294.

34. Ha SA, Shin SM, Namkoong H, Lee H, Cho GW, Hur SY, Kim TE, Kim JW. Cancerassociated expression of minichromos-ome maintenance 3 gene in several human cancers and its involvement in tumorigenesis. CCR. 2004; 10: 8386-8395.

35. Manchano A, Bologna R, Toussaint S, Vega M, González J. Expression of E-cadherin,

36. syndecan 1, Ki-67, and maintenance minichromosome 3 in tissue lesions of actinic prurigo obtained by incisional biopsy. Indian Journal of pathology and microbiology. 2018; 61(2): 225227.

37. Bhasin M, Reinherz EL, Reche PA. Recognition and classification of histones using support vector machine. Journal of Computational Biology. 2016; 13(1): 102-120.

38. Rosenfeld JA, Wang Z, Schones DE, Zhao K, DeSalle R, Zhang MQ. Determination of enriched histone modifications in non-genic portions of the human genome. BMC Genomics. 2009; 10: 143-154.

39. Carey DJ, Stahl RC, Tucker B, Bendt KA, Cizmeci-Smith G. Aggregation-induced association of syndecan - 1 white microfilaments mediated by the cytoplasmic domain. Exp Cell Res. 1994; 214: 12-21.

40. Soukka T, Pohjola J, Inki P, Happonen RP. Reduction of syndecan-1 expression is associated with dysplastic oral epithelium. J Oral Pathol Med. 2000; 29(7): 308-313.

41. Zink LM, Hake SB. Histone variants: nuclear function and disease. Curr Opin Genet Dev. 2016; 37: 82-89.

Vanesa Pereira: vanesapereira91@hotmail.com 